\title{
Design Principle for Fabrication of InP Based Radial Junction Nanowire Solar Cells Using an Electron Selective Contact
}

\author{
Vidur Raj, Member, IEEE, Lan Fu, Member, IEEE, Hark Hoe Tan, Fellow, IEEE, Chennupati \\ Jagadish, Fellow, IEEE
}

\begin{abstract}
Nanowire solar cells hold several advantages over planar solar cells such as reduced reflection, facile strain relaxation, extreme light trapping, increased defect tolerance, etc... However, due to their large surface-to-volume ratio nanowires tend to have very low effective minority carrier lifetime. To overcome this issue a radial junction solar cell was proposed. However, in experimental realization, the efficiency of a radial junction solar cell remains significantly lower than its axial counterpart. This is mainly because of the inability to simultaneously control the doping in both the core and the shell while maintaining low defect density at the interface. To overcome the above-mentioned issues, we propose and simulate a core-shell heterojunction solar cell using p-InP as a core material and ITO/ZnO as a shell material, respectively. Using FDTD simulations, we show that use of an oxide coating over InP core can significantly increase the absorption in InP nanowire arrays, and for an optimized thickness of oxide layer, InP consumption can be reduced by as much as $\mathbf{4}$ folds without sacrificing the ideal short circuit current. In addition, our device simulation results show that even for a core minority carrier lifetime of $50 \mathrm{ps}$, an efficiency of $23 \%$ can be obtained if both core and shell can be heavily doped while maintaining an interface recombination velocity of less than $10^{4} \mathrm{~cm} / \mathrm{s}$. Finally, we discuss how the proposed device structure can reduce the fabrication complexities related to epitaxial homojunction/heterojunction core-shell solar cell structure while achieving a high efficiency under optimized conditions.
\end{abstract}

Index Terms - Nanowire Solar Cell, Optoelectronic Simulation, III-V Photovoltaics, FDTD, TCAD

\section{INTRODUCTION}

$\mathrm{N}$ ANOWIRE solar cells have emerged as an efficient and cost-effective alternative to planar devices [1-4]. Nanowire structures show high absorption and better antireflection properties when compared to their planar counterparts and can thus outperform a planar/thin film device in terms of carrier generation per unit volume of the material used [1-7]. In addition, nanowire architecture allows the growth

This research is supported by the Australian Research Council.

(Corresponding Author: Vidur Raj and Hark Hoe Tan)

The Authors are with the Department of Electronic Materials Engineering, Research School of Physics and Engineering, The Australian National University, Canberra, ACT 2601, Australia (e-mail: vidur.raj@anu.edu.au; lan.fu@anu.edu.au; hoe.tan@anu.edu.au; chennupati.jagadish@anu.edu.au.) of high efficiency III-V materials (e.g., InP/GaAs) on earth abundant/inexpensive cheap substrates (e.g., silicon, glass, etc...) $[4,8,9]$. However, the high density of surface states along with a large surface-to-volume ratio can drastically reduce minority carrier diffusion lengths and lifetimes in nanowires [5, $10,11]$. Consequently, novel device structures such as a radial junction architecture has been proposed to achieve high efficiency $[12,13]$.

Schematic representation of an axial and a radial junction solar cell is shown in Figure 1(a) and 1(b), respectively. For efficient light absorption, the length of nanowire should be longer than the absorption depth (denoted by $1 / \alpha$ in Figure 1) [12]. Similarly, for efficient charge collection, the distance between the points of minority carrier generation to the $p-n$ junction area should be less than the diffusion length (denoted as $L_{n}$ and $L_{p}$ in Figure 1) [12]. In a radial junction solar cell (shown in Figure 1(b)), incident light can be absorbed in a relatively long axial direction of the nanowire while the electrons and holes are collected in the radial direction that is only tens of nanometers thick. A radial junction solar cell structure is more suitable when the absorbing material has a smaller diffusion length (lower lifetime). After the seminal work of Kayes et.al. [12, 13], several of the authors have reported radial homo- and heterojunction solar cells based on $\mathrm{Si}, \mathrm{GaAs}, \mathrm{ZnSe}, \mathrm{Sb}_{2} \mathrm{~S}_{3}$ etc., both theoretically and experimentally [12, 14-18]. However, a detailed optoelectronic simulation on use of an electron selective contact for heterojunction solar cells based on InP, remain unexplored.

Indium Phosphide (InP) is a direct band gap semiconductor with much higher absorption coefficient compared to indirect semiconductors such as silicon, yet the cost of InP solar cells remains prohibitive for terrestrial applications, mostly due to high material cost [4]. To overcome this limitation, InP nanowire-based solar cells are being aggressively pursued by various research groups because of the unique properties offered by nanowires as mentioned above $[2,4,5,12,18]$. Although efficiency as high as $17.8 \%$ has been reported for InP

An electronic copy of the supporting information is available online or from the author.

Color versions of one or more of the figures in this paper are available online at http://ieeexplore.ieee.org 
nanowire solar cells, it remains substantially below to the best reported planar efficiency that is $29.1 \pm 0.6 \%$ [19, 20]. It is predicted that core-shell design can significantly improve nanowire solar cell efficiency $[12,13]$, however, in practice the performance of III-V nanowire solar cells with radial geometry is below expectation because of following reasons [5, 21]. In general, III-V core-shell nanowire solar cell structures are grown epitaxially $[4,5]$. In an epitaxially grown core-shell structure, accurate control of doping in both core and shell with a defect free interface is difficult because of the out diffusion of dopants during shell growth along with other problems such as facet dependent dopant incorporation, unwanted shell growth, and the difficulty in dopant incorporation especially for nanowires grown by the vapor-liquid-solid (VLS) mechanism [5, 21-23]. Moreover, core-shell nanowire structure starts with the growth of a core segment, during which defects and impurities can be incorporated on the sidewall of the core [2426], subsequent growth of the shell over a defective core surface leaves the impurities and defects trapped at the $p-n$ junction interface, leading to inferior performance of the solar cell [24]. Therefore, to realize high efficiency core-shell nanowire solar cells, an alternative approach is needed.

Most recently, passivating charge carrier selective contacts have shown huge potential to overcome the optoelectronic losses of conventional p-i-n homojunction solar cells [27]. In fact, most of the high efficiency thin film and silicon solar cells incorporate heterojunction for improved performance [26-30]. Conventionally, epitaxial III-V based thin film solar cells used wide band gap III-V materials as window layers for passivation and charge carrier selectivity [31, 32]. However, for nanowire core shell structure, growth of a doped wide band gap epitaxial shell over III-V core can be challenging because of reasons mentioned above. An alternative to this problem is the use of a wide band gap metal oxides which has successfully been implemented in III-V solar cells to achieve high open circuit voltage and efficiency [33, 34]. Until now, a detailed optoelectronic analysis of nanowire core-shell heterojunction solar cell based on these charge carrier selective contacts has not been performed.

In this paper, we perform a coupled optoelectronic simulation on an ITO/ZnO/p-InP heterojunction solar cell, where zinc oxide $(\mathrm{ZnO})$ is used as a passivating electron selective contact for p-type InP nanowires. We show that use of ITO/ZnO shell over InP can significantly improve the absorption efficiency of these cells while achieving an efficiency of more than $25 \%$ under optimum conditions. Not to mention, even for core minority carrier lifetime as low as $50 \mathrm{ps}$, an efficiency of $~ 23$ $\%$ can be achieved if a core doping of more than $1 \times 10^{18} \mathrm{~cm}^{-3}$ can be obtained while maintaining a low surface recombination velocity of $10^{4} \mathrm{~cm} / \mathrm{s}$. Furthermore, we show that the proposed heterojunction solar cell structure can simplify several of the growth complexities associated with core-shell nanowires grown either by the VLS or selective area epitaxy techniques.

\section{MODELING METHODS}

\section{A. FDTD Simulation}

To study the optoelectronic behaviors of $\mathrm{ZnO} / \mathrm{InP}$ core-shell heterojunction solar cells, we firstly perform an optical simulation using Lumerical's finite-difference time-domain (FDTD) module. It is a commercial-grade simulator that solves the electromagnetic equations in time domain using the gridbased (Yee-cells) differential numerical modelling methods (finite difference methods) [35]. FDTD is a state-of-the-art method for solving Maxwell's equations in complex geometries to calculate the $\mathrm{E} \rightarrow(\mathrm{r}, \omega)$ (electric field) and $\mathrm{H} \rightarrow(\mathrm{r}, \omega)$ (magnetic field) at all points within the computational domain, which can then be used to calculate the absorbed power as a function of space and angular frequency using the following equation $[36,37]$

$$
P_{a b s}(\vec{r}, \omega)=\frac{1}{2} \omega|E(\vec{r}, \omega)|^{2} \operatorname{Im}\{\epsilon(\vec{r}, \omega)\}
$$

Where, $P_{a b s}$ is the power absorbed, $\omega$ is the angular frequency, $|E|^{2}$ is the electric field intensity, and $\operatorname{Im}(\epsilon)$ is the imaginary part of the permittivity. Moreover, in the ideal case, it can be assumed that each absorbed photon excites an ehp (electron-hole pair) and the generation rate in such cases is equivalent to photon absorption rate. Then, the generation rate, $G(\vec{r})$ for solar spectrum can be calculated by normalising the absorbed power to solar spectrum as

$$
G(\vec{r}) \approx \int \frac{P_{a b s}(\vec{r}, \omega) \cdot I_{\text {solar }}(\omega)}{I_{\text {source }}(\omega)} d \omega
$$

The ideal short circuit current density can then be calculated using

$$
J_{s c(\text { ideal })}=\frac{e \int G(\vec{r}) \cdot d r}{A}
$$

Where, $e$ is the electronic charge and $A$ is area of the solar cell. We use ideal short circuit current calculated using equation 3 as a performance parameter to study the effect of the shell over InP core. Figure 2(a) shows a 3-D model of simulated nanowire array of ITO/ZnO/p-InP core-shell heterojunction solar cell. In the model we incorporate, InP nanowire as core, $\mathrm{ZnO}$ as shell and a layer of indium tin oxide (ITO) as a transparent contact layer to improve the electrical characteristics. To perform FDTD simulations, the refractive index (n) and the extinction coefficient $(\mathrm{k})$ of $\mathrm{InP}$ was obtained from the software (Lumerical FDTD) itself. However, the $\mathrm{n}$, $\mathrm{k}$ values for $\mathrm{ZnO}$ and ITO was measured using ellipsometer, in a separate but related work. The experimental values were then fitted using Lumerical's in-built analytical model and the best fit $n$, $k$ values were used for performing the simulation. Figure $\mathrm{S} 1$ of the supplementary section shows experimental $\mathrm{n}$, $\mathrm{k}$ values of ITO and $\mathrm{ZnO}$ fitted using Lumerical's in-built analytical model. In Figure 2(b), an enlarged schematic of $y$-z cross sectional view of core-shell heterojunction solar cell is illustrated along with the material specification and geometrical parameters. Previous reports on simulation of optical absorption in InP nanowires show that the optimum pitch for a nanowire solar cell should be about $480 \mathrm{~nm}[4,38]$. Hence, we fixed the pitch of nanowire as $480 \mathrm{~nm}$ and the thickness of the shell, R_Shell, was varied from 0 to $180 \mathrm{~nm}$. The total thickness of the shell is a combined 
thickness of $\mathrm{ZnO}$ and ITO. For simplicity, we fix the thickness of $\mathrm{ZnO}\left(\mathrm{R} \_\mathrm{ZnO}\right)$ at $10 \mathrm{~nm}$ and optimize the thickness of ITO (R_ITO) to achieve a high ideal short circuit current. Increasing the thickness of ITO is reasonable considering that a thicker ITO will be required to achieve low series resistance in practical situations. In addition, a sufficiently thick $\mathrm{ZnO}(10 \mathrm{~nm})$ was required to reduce the divergence problem during electronic simulations. Nonetheless, the effect of $\mathrm{ZnO}$ thickness on the optical and electronic performance of ITO/ZnO/p-InP is shown in Figure S2 and Figure S3 of the supplementary section. In addition, while optimizing the thickness of the ITO for a given core radius, the total radius of core and shell is maintained at less than the half of the pitch, i.e. 2 x (R_Core + R_Shell) < Pitch. Another important geometrical parameters such as thickness of the substrate and the silicon dioxide is given as T_Sub and T_SiO2, respectively.

\section{B. Device Simulation}

In order to perform a 2-D device simulation, the optical generation rate obtained using Lumerical FDTD simulator is transferred to the Lumerical charge solver which is a powerful TCAD (Technology Computer-Aided Design) semiconductor device simulation software with ability to self-consistently solve the drift-diffusion, continuity and Poisson's equations in 2-D and 3-D [35]. Detailed mathematical and physics formalism of device charge transport related to solar cells is given in supplementary section of the paper. Based on initial FDTD optimization we perform device simulations for an InP core of $80 \mathrm{~nm}$ radius with $10 \mathrm{~nm}$-thick $\mathrm{ZnO}$ and $80 \mathrm{~nm}$-thick ITO, respectively. A $200 \mathrm{~nm}$-thick $\mathrm{SiO}_{2}$ is used to isolate the electronic contribution from the substrate. Also, a heavily pdoped $\left(1 \times 10^{19} \mathrm{~cm}^{-3}\right)$ substrate of thickness $200 \mathrm{~nm}$ is assumed during device simulation to reduce the divergence problems. Some of the important material parameters which were kept constant during device simulation is given in table ST1 of the supplementary section. To make our electronic simulation more realistic we vary the mobility of InP nanowire core based on the work of Joyce et al. [39] who have shown that the carrier mobility of undoped InP nanowire can be as low as $700 \mathrm{~cm}^{2} \mathrm{~V}^{-}$ ${ }^{1} \mathrm{~s}^{-1}$ as compared to bulk or high quality epilayer InP, which can reach as high as $6000 \mathrm{~cm}^{2} \mathrm{~V}^{-1} \mathrm{~s}^{-1}$. So, during electrical simulation we use the maximum lattice scattering electron mobility of 700 $\mathrm{cm}^{2} \mathrm{~V}^{-1} \mathrm{~s}^{-1}$ and hole mobility of $100 \mathrm{~cm}^{2} \mathrm{~V}^{-1} \mathrm{~s}^{-1}[39,40]$, and use the built-in Caughey-Thomas model to account for the influence of impurities on carrier mobility. More details on the Caughey-Thomas model can be found in the supplementary section. Nevertheless, as shown earlier in Figure 1, a core-shell solar cell structure is mainly desirable when the minority carrier lifetime in core is low, so we limit our simulations to a low minority carrier lifetime ranging from 50 ps to $1 \mathrm{~ns}$. Finally, we include surface recombination into our simulation and vary the surface recombination velocity from $10^{3} \mathrm{~cm} / \mathrm{s}$ to $10^{7} \mathrm{~cm} / \mathrm{s}$. For both bulk and surface recombination a single trap level at the mid bandgap was assumed [12, 13].

\section{RESULTS}

\section{A. Optimization of Oxide Thickness}

Optoelectronic simulation of ITO/ZnO/p-InP core-shell heterojunction solar cell started with the optimization of oxide thickness for a wide range of core radii. We use ideal short circuit current $\left(\mathbf{J}_{\text {sc(ideal) }}\right)$ calculated using equation (3) as a performance parameter to optimize the oxide shell thickness for different core radii. To avoid any contribution from ITO or $\mathrm{ZnO}$, the integration in equation (3) was performed only within the InP nanowire boundary, i.e. only the charge carriers generated inside $\mathrm{InP}$ nanowire is contributing to $\left(\mathrm{J}_{\mathrm{sc}}(\right.$ ideal) $)$. The $\left(\mathrm{J}_{\text {sc(ideal) }}\right)$ as a function of shell thickness (R_Shell) and core radius (R_Core) is given in Figure 2(c). It is quite evident that for all values of $R$ _Core considered here, ( $\mathrm{J}_{\mathrm{sc} \text { (ideal })}$ ) increases with R_Shell when compared to uncoated nanowires. However, this increase in $\left(\mathrm{J}_{\mathrm{sc} \text { (ideal) }}\right)$ with increased $\mathrm{R} \_$Shell is not monotonic, and it shows a small drop after reaching a maximum value for optimum shell thickness. The increase in ( $\left.\mathrm{J}_{\mathrm{sc} \text { (ideal }}\right)$ of $\mathrm{InP}$ core in presence of an oxide shell is because of an enhanced absorption in the InP nanowire with an oxide coating as described later. Moreover, for smaller core radius the effect of shell thickness is more pronounced as compared to wider core. For example, for $50 \mathrm{~nm}$ core radius the difference between bare InP and InP coated with optimized shell is $\sim 7 \mathrm{~mA} / \mathrm{cm}^{2}$, whereas, as the core radius increases to $80 \mathrm{~nm}$ this difference is only $\sim 4 \mathrm{~mA} / \mathrm{cm}^{2}$. As the thickness of the core is increased further the difference between bare and oxide coated InP reduces to $\sim 2 \mathrm{~mA} / \mathrm{cm}^{2}$. We find that there is a fairly broad range of oxide thickness which is optimum for most of the InP core radius.

It has been shown both experimentally and theoretically, that reduced screening of incident electric field [41] and an optical antenna effect [41-44] causes an increased absorption in absorbing nanowire core coated with a non-absorbing oxide layer. Similar to previous reports, we also see increased confinement of electric field in oxide coated nanowires in comparison to bare InP nanowires. Because, this topic has previously been discussed in detail by several of the authors [41-44], herein, we restrict our study to InP core of radius 80 $\mathrm{nm}$, with and without $90 \mathrm{~nm}$ of oxide shell.

Comparative absorption spectra an of InP nanowire of $80 \mathrm{~nm}$ radius with and without a $90 \mathrm{~nm}$-thick oxide shell is shown in Figure 3(a). Along with comparative absorption spectra in Figure 3(a), a 2-D generation plot for bare and oxide coated InP nanowire is shown in Figure 3(b) and 3(c), respectively. When plotted on same scale, InP nanowire coated with oxide (Figure $3(\mathrm{c}))$ shows a maximum generation rate of $8 \times 10^{22} \mathrm{~cm}^{-3} \mathrm{~s}^{-1}$, whereas, the maximum generation rate in uncoated InP ((Figure $3(b))$ nanowire remains at $6 \times 10 \mathrm{~cm}^{-3} \mathrm{~s}^{-1}$. Higher generation rate in an oxide coated InP nanowire is a result of improved absorption in oxide coated InP in comparison to bare InP. To better understand increased absorption and generation in the oxide coated InP nanowire, we simulated the electric field distributions for both of the structures (bare and oxide coated InP) at five different wavelengths. The normalized electric field distributions in a bare and oxide coated InP are shown in Figures 3(d-h) and 3(i-m), respectively. It is quite apparent that the electric field distribution in bare InP at 400, 500 and 600 
$\mathrm{nm}$, is distributed within the area between the nanowires with only small amounts of the electric field confined within the nanowire itself. On the contrary, electric field distribution in an oxide coated InP is concentrated at the top segment of the nanowire and reduces significantly towards the base of the nanowire, which suggest that an oxide coated nanowire shows higher absorption than that of bare InP. This is in agreement with absorption spectra (shown in Figure 3(a)), where the use of an oxide layer increases the light absorption in 400-600 nm wavelength region. At the wavelength of $700 \mathrm{~nm}$, both bare (figure 3(g)) and oxide coated InP (figure 3(1)) show high electric field confinement and therefore high absorption. However, comparing the electric field of bare (Figure 3(h)) and coated InP nanowire (Figure 3(m)) at the wavelength of 800 $\mathrm{nm}$, it is quite clear that the residual electric field below $1 \mu \mathrm{m}$ length in bare InP is higher than the oxide coated InP nanowire. For example, the residual electric field in bare InP at $0.75 \mu \mathrm{m}$ (from base) is 0.62 on the normalized scale, whereas, the residual electric field in oxide coated $\mathrm{InP}$ at $0.75 \mu \mathrm{m}$ (from base) is 0.38 . This shows better confinement of electric field (higher absorption) in nanowires coated with oxide in comparison to bare InP at $800 \mathrm{~nm}$ wavelength. Nonetheless, both bare and oxide coated InP nanowires absorbs most of the $800 \mathrm{~nm}$ wavelength before $2 \mathrm{um}$ along their length and therefore shows no difference in absorption plotted in figure 2(a).

Another important parameter to describe the nanowire array is its geometrical fill factor given as following:

$$
\text { Geometrical Fill Factor }(G F F)=\frac{\pi\left(R_{\text {Core }}\right)^{2}}{(\text { Pitch })^{2}}
$$

Several authors have previously reported that to achieve $\mathbf{J}_{\mathrm{sc}}$ of more than $32 \mathrm{~mA} / \mathrm{cm}^{2}$, the InP nanowire GFF should be more than 0.17 [38, 45, 47]. However, as shown in Figure 2(c), using an oxide shell of $90 \mathrm{~nm}$ on an $80 \mathrm{~nm}$ InP core, a $J_{\text {sc }}$ of 32.432 $\mathrm{mA} / \mathrm{cm}^{2}$ (marked using black square in Figure 2(c)) can be achieved which corresponds to an absorber GFF of less than 0.09 (see upper Y-axis). In volumetric terms, use of an optimal oxide shell over InP core can reduce the material requirement by almost 4 times. Furthermore, it has recently been reported that for a fixed geometrical fill factor both square and hexagonal arrays of nanowires give similar $\mathrm{J}_{\mathrm{sc}}[45,46]$, therefore, we would expect that most of results obtained here should also be applicable to hexagonal arrays.

\section{B. Electrostatics and Band Diagram}

The band diagram of an ITO/ZnO/p-InP heterojunction solar cell at thermal equilibrium is shown in Figure 4. To draw the band diagram in Figure 4, a p-type core doping of $1 \times 10^{18} \mathrm{~cm}^{-3}$ and p-type substrate doping of $1 \times 10^{19} \mathrm{~cm}^{-3}$ was assumed, whereas, the n-type doping in $\mathrm{ZnO}$ and ITO doping was fixed at $1 \times 10^{18} \mathrm{~cm}^{-3}$ and $1 \times 10^{20} \mathrm{~cm}^{-3}$, respectively. Band diagram plotted in Figure 4 shows that $\mathrm{ZnO}$ forms a type-II heterojunction with p-type InP with a small conduction band offset and a large valence band offset. Furthermore, a heavily doped ITO layer over $\mathrm{ZnO} / \mathrm{InP}$ ensures that maximum band bending happens in the absorber layer.

Most recently, Chen et al. have shown that in a well passivated $\mathrm{p}-\mathrm{n}$ homojunction InP nanowire solar cell, contact leakage (flow of minority carriers towards the metal contact instead of p-n junction) limits the efficiency by reducing the open circuit voltage $\left(\mathrm{V}_{\mathrm{oc}}\right)$ and fill factor (FF) [48]. In the proposed heterojunction solar cell, contact leakage can be reduced to minimum because $\mathrm{ZnO}$ forms a type-II heterojunction with p-type InP, with a small conduction band offset which allows the flow of electrons, and a large valence band offset which restricts the flow of holes (minority carriers) to the surface. Moreover, a wide band gap electron selective contact, such as $\mathrm{ZnO}$ maintains an extremely low minority carrier conductivity under illumination because of low absorption coefficient and low intrinsic carrier concentration $[33,49]$. In other words, a large valence band offset and an extremely low minority carrier conductivity in $\mathrm{ZnO}$ can significantly reduce the flow of minority charge carriers to the front contact thereby reducing the surface recombination in the charge carrier selective layer and recombination at the oxide/metal (front contact) interface which in turn leads to more efficient solar cell operation [33, 50, 51].

Another important parameter of a nanowire solar cell is the electric field distribution at the $\mathrm{p}-\mathrm{n}$ junction. An electric field plot of the heterojunction solar cell for different $\mathrm{p}$-type core doping concentrations is shown in Figure 5(a). It is quite clear from Figure 5(a) that increasing the doping concentration of $\mathrm{p}$ type InP core from $1 \times 10^{16} \mathrm{~cm}^{-3}$ to $1 \times 10^{19} \mathrm{~cm}^{-3}$, increases the radial built-in electric field, which in turn increases the probability for the minority carriers (electrons) to diffuse more efficiently to the depletion region. A corresponding 2-D plot for electric field as a function of radial distance is shown for different p-type core doping concentrations in Figure 5(b-e). The 2-D electric field plots show that a doping concentration of more than $1 \times 10^{17} \mathrm{~cm}^{-3}$ is required to achieve a higher built-in electric field in radial direction as compared to the built-in electric field at substrate-core interface. The reason for this becomes clearer when we plot the effective charge carrier concentration at thermal equilibrium as a function of radial distance for different p-type core doping concentrations as shown in Figure 5 (f-i). It is quite evident that at a low core doping of $1 \times 10^{16} \mathrm{~cm}^{-3}$, the core is fully depleted and behaves as a very lightly n-type material to form a junction at the substratecore interface and not at the $\mathrm{ZnO} / \mathrm{InP}$ interface (because $\mathrm{ZnO}$ is also n-type). But as the p-type doping in the core increases to $1 \times 10^{17} \mathrm{~cm}^{-3}$, the core starts behaving like an intrinsic region with low p-type doping. With further increase in doping, the core becomes more and more $\mathrm{p}$-type and a more effective $\mathrm{p}-\mathrm{n}$ junction is formed at the $\mathrm{ZnO} / \mathrm{InP}$ interface leading to an electric field as high as $1.2 \times 10^{8} \mathrm{~V} / \mathrm{m}$. Similar results have been reported for homojunction GaAs core-shell solar cell using simulations [52].

\section{Effect of Doping and Lifetime}

In this section, we study the effect of doping and minority carrier lifetime on different performance parameters of the heterojunction solar cell. As discussed earlier, a core-shell solar cell structure is more desirable when the minority carrier lifetime in the core is low, so we limit our study to low minority carrier lifetimes ranging from 50 ps to $1 \mathrm{~ns}$. Different solar cell 
performance parameters such as efficiency, open circuit voltage $\left(\mathrm{V}_{\mathrm{oc}}\right)$, short circuit current density $\left(\mathrm{J}_{\mathrm{sc}}\right)$, and fill factor (FF) are plotted in Figure 6 (a-d), respectively as a function of core minority carrier lifetime and core doping density. To simultaneously study the effect of lifetime and doping, the surface recombination velocity at $\mathrm{ZnO} / \mathrm{InP}$ interface was not included during the simulation. However, surface recombination velocity of $10^{7} \mathrm{~cm} / \mathrm{s}$ was assumed for both the top and bottom metal contacts. Figure 6(a) shows that to achieve an efficiency $>25 \%$ a p-type doping higher than $1 \times 10^{18}$ $\mathrm{cm}^{-3}$ is required, which agrees well with the simulated electric field profile shown in Figure 5. Moreover, an increase in core lifetime increases the efficiency for all doping ranges mainly because of reduced Shockley-Read-Hall (SRH) recombination in the bulk. A higher core doping is required to achieve high efficiency because higher doping leads to a high $\mathrm{V}_{\mathrm{oc}}$ (Figure $6(\mathrm{~b})$ ) and high fill factor (Figure 6(d)). However, a high $\mathrm{J}_{\mathrm{sc}}$ of $>30 \mathrm{~mA} / \mathrm{cm}^{2}$ can be achieved for a wide range of doping concentration with the maximum $\mathrm{J}_{\mathrm{sc}}$ achieved at $\sim 1 \times 10^{17} \mathrm{~cm}^{-3}$ core doping. Higher $\mathrm{V}_{\mathrm{oc}}$ is achieved for higher core doping because of a larger electron and hole quasi-fermi level splitting with increased doping concentration as shown in Figure S(4) of the supplementary section. A larger electron and hole quasifermi level splitting with increased doping concentration is a result of reduced recombination with increased doping. Further, a decrease in recombination with increased doping is a combined effect of reduced depletion region width (i.e. reduced recombination in depletion region) as well as an enhanced builtin electric field (i.e. efficient charge carrier extraction). Moreover, at lower doping the core is depleted of carriers (Figure 5(f) and 5(g)) which significantly reduces the conductivity of the core and increases the overall series resistance, thereby leading to a low FF. Thus, an optimum $\mathrm{V}_{\text {oc }}$ and FF can be achieved only for doping level higher than $1 \times 10^{18}$ $\mathrm{cm}^{-3}$. $\mathrm{J}_{\mathrm{sc}}$ is maximum at a doping level of $\sim 1 \times 10^{17} \mathrm{~cm}^{-3}$ because the space charge region expands all the way into the core (see Figure 5(a) and 5(c)) such that almost all the carriers generated in the core reach the junction before recombination. In absence of surface recombination at $\mathrm{ZnO} / \mathrm{InP}$ interface, a core minority carrier lifetime of more than $1 \mathrm{~ns}$ for a core doping of more than $5 \times 10^{18} \mathrm{~cm}^{-3}$ can achieve an efficiency of $\sim 30 \%$, which is about the predicted Shockley-Queisser limit for InP.

\section{Effect of Surface Recombination Velocity (at ZnO/InP interface) when $\tau_{n}=1 \mathrm{~ns}$}

As shown earlier, in absence of surface recombination even for a core lifetime as low as $200 \mathrm{ps}$, a high efficiency of $>25 \%$ can be achieved for a core doping of more than $1 \times 10^{18} \mathrm{~cm}^{-3}$. However, core-shell solar cell structures are more susceptible to surface recombination in comparison to bulk recombination process in the core itself. Next, we simulate the effect of surface recombination velocity (SRV) at the $\mathrm{ZnO} / \mathrm{InP}$ interface as function of doping. We study the effect of SRV for a core minority carrier lifetime of $50 \mathrm{ps}$ and $1 \mathrm{~ns}$. The simulated solar cell performance parameters such as efficiency, $\mathrm{V}_{\mathrm{oc}}, \mathrm{J}_{\mathrm{sc}}$ and $\mathrm{FF}$ for $1 \mathrm{~ns}$ carrier lifetime is plotted in Figure 7 (a-d), respectively, as a function of different core doping concentrations and SRVs.
As expected, an increase in SRV significantly degrades the solar cell efficiency for all values of core doping densities. For example, for a core doping of $1 \times 10^{18} \mathrm{~cm}^{-3}$, the efficiency of the heterojunction solar cell reduces from $\sim 28.2 \%$ to $16.6 \%$, for an increase in SRV from $1000 \mathrm{~cm} / \mathrm{s}$ to $10^{7} \mathrm{~cm} / \mathrm{s}$. This decrease in efficiency is mainly because of significant degradation in the $\mathrm{V}_{\mathrm{oc}}$ and $\mathrm{FF}$ of the ITO/ZnO/p-InP solar cell with increased SRV.

To understand the degradation of $\mathrm{V}_{\mathrm{oc}}$ with increased $\underline{\mathrm{SRV}}$, we can write $\mathrm{V}_{\text {oc }}$ as:

$$
V_{o c} \approx\left(\frac{n k T}{q}\right) \ln \left(\frac{J_{L}}{J_{0}}\right)
$$

Here, $\mathrm{J}_{\mathrm{L}}$ and $\mathrm{J}_{0}$ are the light generated current density and dark saturation current density, respectively. The above expression shows that the $\mathrm{V}_{\text {oc }}$ can be increased by increasing light generated current and/or by decreasing the dark saturation current. In general, light generated current remains almost similar while dark current can vary by several orders of magnitude. Furthermore, the dark current under forward bias is entirely due to recombination and a higher dark current means a higher recombination in the device. Dark current density vs voltage plots at different SRV for different core doping densities are shown in Figure S5 of the supplementary section. As expected, an increase in SRV increases dark current for almost all densities of core doping used during simulation, leading to a lower $\mathrm{V}_{\mathrm{oc}}$. At the same time, a higher core doping leads to orders of magnitude lower dark current (shown in Figure S5 (a-e) of the supplementary section) as compared to lower core doping densities, for a given SRV. This shows that for a given SRV, the recombination is remarkably lower at higher core doping (more than $1 \times 10^{18} \mathrm{~cm}^{-3}$ ) compared to lower core doping densities. This is further confirmed by plotting the $\mathrm{SRH}$ recombination (at $\mathrm{SRV}=10^{5} \mathrm{~cm} / \mathrm{s}$ ) at $\mathrm{V}_{\mathrm{oc}}$ as a function of radial distance (see Figure S6 of the supplementary section) for different core doping densities. It is quite evident that the recombination rate reduced by almost 3 orders of magnitude as the core doping density increases from $1 \times 10^{16} \mathrm{~cm}^{-3}$ to $1 \times 10^{18}$ $\mathrm{cm}^{-3}$. In addition, at doping level below $1 \times 10^{18} \mathrm{~cm}^{-3}$, most of the recombination happens in depletion region, which significantly degrades the $\mathrm{V}_{\mathrm{oc}}$.

Moreover, a higher fill factor for higher core doping stems from a combined effect of reduced recombination (low $\mathbf{J}_{0}$ ) and improved extraction of charge carriers (high electric field). In comparison to $\mathrm{FF}$ and $\mathrm{V}_{\mathrm{oc}}, \mathrm{J}_{\mathrm{sc}}$ is less dependent on core doping and/or SRV, and a $\mathbf{J}_{\mathrm{sc}}>30 \mathrm{~mA} / \mathrm{cm}^{2}$ can be obtained for core doping more than $1 \times 10^{17} \mathrm{~cm}^{-3}$ even for SRV as large as $1 \times 10^{7}$ $\mathrm{cm} / \mathrm{s}$. However, for a core doping below $6 \times 10^{16} \mathrm{~cm}^{-3}, J_{\mathrm{sc}}$ degrades significantly for SRV $>10^{5} \mathrm{~cm} / \mathrm{s}$. $\mathrm{J}_{\mathrm{sc}}$ doesn't degrade significantly with increased SRV or decreased lifetime, because in a core-shell structure the diffusion length of minority carriers remains larger than the radius of core which ensures that most of the carriers are extracted before they can recombine.

\section{E. Effect of Surface Recombination Velocity (at ZnO/InP interface) when $\tau_{n}=50 \mathrm{ps}$}

When the core minority carrier lifetime is set to $50 \mathrm{ps}$, the effect of SRV on solar cell efficiency, $V_{\text {oc }}, J_{\text {sc }}$ and, FF follow 
similar trend to that of $1 \mathrm{~ns}$, as shown in Figure 8(a-d). However, the maximum achievable efficiency reduces to 23.2 $\%$ in comparison to $28.6 \%$ achieved when core minority carrier lifetime was set at $1 \mathrm{~ns}$. As expected, lower lifetime combined with higher SRV gives lower efficiency mainly because of increased SRH recombination, which degrades both $\mathrm{V}_{\text {oc }}$ and FF. Here too, a heavily doped core is found to be more suitable for achieving high efficiency. Also, an efficiency of almost $23 \%$ can be obtained for core lifetime as low as $50 \mathrm{ps}$ and SRV of $10^{4} \mathrm{~cm} / \mathrm{s}$, when core doping is more than $1 \times 10^{18} \mathrm{~cm}^{-3}$. However, for the proposed heterojunction nanowire solar cells, superposition principle breaks (see Figure S7 of supplementary section) even for low SRV when the minority carrier lifetime is extremely low $(50 \mathrm{ps})$, most probably due to high recombination in the depletion region [46]. Nevertheless, most recently, Attu et al. have shown that the efficiency of a p-n junction nanowire in axial configuration reduces to $15 \%$ for lifetime less than $300 \mathrm{ps}$, whereas, for current device structure an efficiency of $23 \%$ can be achieved even for a nanowire lifetime of $50 \mathrm{ps}$ [46]. This clearly shows the benefit of the current solar cell structure over an axial p-n junction.

\section{DISCUSSION}

In general, III-V nanowire lifetimes are within few ns to several ps limit [5, 10-11, 40]. Therefore, for III-V nanowire solar cells, a radial core-shell junction design is expected to be more efficient than an axial junction design $[5,12,13]$. However, in practice reports on high efficiency III-V nanowire solar cells are mainly limited to axial junction design [5]. Although no detailed investigation is available, the efficiency of epitaxially grown III-V core-shell solar cell structure appears to be limited by interface quality between the p-doped and the n-doped regions and inability to controllably and uniformly dope both the core and shell $[21,23,24,26,53]$. In this section, we discuss the significance and practicality of proposed InP nanowire heterojunction solar cell as an alternative to core-shell structures grown epitaxially.

One of the major advantages of ITO/ZnO/p-InP device structure is that only p-type InP has to be grown epitaxially (by Metal Organic Chemical Vapor Deposition (MOCVD) or Molecular Beam Epitaxy (MBE)) and the outer shell ( $\mathrm{ZnO} / \mathrm{ITO})$ can then be deposited using Atomic Layer Deposition (ALD) or other conformal deposition techniques. Because, in an ITO/ZnO/p-InP solar cell structure, the bulk properties of shell is not significantly influenced by the underlying core, the shell can be optimized separately to achieve optimum electronic behaviour. In comparison, when both core and shell are grown epitaxially, the quality of the shell depends heavily on the core $[16,26,53]$. In addition, for an epitaxial core-shell homojunction/heterojunction solar cell structure, both the core and shell have to be grown at higher temperatures (temperature $>600{ }^{\circ} \mathrm{C}$ ) leading to unwanted out diffusion of dopants from core to shell and vice-versa [5, 23, 26]. This cross diffusion of dopants can significantly degrade the electronic properties of the core-shell. On the other hand, for fabrication of the proposed heterojunction solar cell, only $\mathrm{InP}$ core has to be grown at higher temperatures while the oxide shell can be deposited at a relatively low temperature $\left(<300^{\circ} \mathrm{C}\right)$ using ALD and/or another conformal/semi-conformal deposition techniques. Thus, the proposed heterojunction solar cell is expected to significantly reduce the out diffusion of dopants from core while maintaining the as-grown quality of nanowires. As shown in previous sections, along with reducing the fabrication complexities, the use of an oxide shell over InP also improves the optical response leading to a higher short circuit current. Furthermore, when compared with previous reports on InP nanowire solar cells $[46,48]$, the simulated device structure seems to be less susceptible to SRV and low core lifetime, at least in higher core doping regimes. However, to achieve a high efficiency, it is extremely desirable to have a surface recombination velocity less than $10^{4} \mathrm{~cm} / \mathrm{s}$, while maintaining a p-type doping more than $1 \times 10^{18} \mathrm{~cm}^{-3}$. For InP nanowires, surface recombination velocity of the order of $10^{4}$ $\mathrm{cm} / \mathrm{s}$ have readily been achieved both in doped and undoped InP nanowires and therefore the proposed structure has true potential for real life implementation [40, 54-55].

\section{CONCLUSION}

In conclusion, the proposed radial $\mathrm{p}-\mathrm{n}$ heterojunction nanowire solar cell has true potential to achieve high efficiency while reducing the cost and fabrication complexities related to epitaxially grown homo/hetero junction core-shell nanowire solar cells. We have shown that use of an optimum thickness of $\mathrm{ZnO} / \mathrm{ITO}$ shell significantly enhances the absorption in an InP nanowire core and the ideal $\mathrm{J}_{\mathrm{sc}}$ can be improved by more than 7 $\mathrm{mA} / \mathrm{cm}^{2}$ for smaller nanowire radius. Moreover, for geometrical fill factor less than 0.09 , an ideal $\mathrm{J}_{\mathrm{sc}}$ of $\sim 32.5$ $\mathrm{mA} / \mathrm{cm}^{2}$ can be realized. In addition to optical simulations, we systematically study the simultaneous effect of doping, lifetime and surface recombination on ITO/ZnO/p-InP heterojunction solar cell. Based on wide range of device simulations, we find that an optimal heterojunction solar cell should have a core doping higher than $1 \times 10^{18} \mathrm{~cm}^{-3}$, and a SRV of less than $10^{5}$ $\mathrm{cm} / \mathrm{s}$. Finally, it is shown that even for core carrier lifetime as low as $50 \mathrm{ps}$, an efficiency of $23 \%$ can be achieved if a core doping of more than $1 \times 10^{18} \mathrm{~cm}^{-3}$ can be achieved while maintaining a low surface recombination velocity of $10^{4} \mathrm{~cm} / \mathrm{s}$.

\section{ACKNOWLEDGMENT}

This research is supported by the Australian Research Council and Australian National Fabrication Facility is acknowledged for access to simulation tools.

\section{REFERENCES}

[1] M. C. Beard, J. M. Luther, and A. J. Nozik, "The promise and challenge of nanostructured solar cells," Nat. Nanotechnology, vol. 9, pp. 951, 2014. DOI: doi.org/10.1038/nnano.2014.292

[2] E. C. Garnett, M. L. Brongersma, Y. Cui, and M. D. McGehee, "Nanowire Solar Cells," Annu. Rev. Mater. Res., vol. 41, no. 1, pp. 269-295, 2011. DOI: doi.org/10.1146/annurev-matsci-062910-100434

[3] E. Garnett, and P. Yang, "Light Trapping in Silicon Nanowire Solar Cells," Nano Lett. , vol. 10, no. 3, pp. 1082-1087, 2010. DOI: $10.1021 / \mathrm{nl} 100161 \mathrm{z}$

[4] J. Wallentin, et al., "InP Nanowire Array Solar Cells Achieving 13.8\% Efficiency by Exceeding the Ray Optics Limit," Science., vol. 339, no. 
6123, pp. 1057, 2013. DOI: 10.1126/science. 1230969

[5] G. Otnes, and M. T. Borgström, "Towards high efficiency nanowire solar cells," Nano Today, vol. 12, pp. 31-45, 2017. DOI: https://doi.org/10.1016/j.nantod.2016.10.007

[6] B. Tian, X. Zheng, T. J. Kempa, Y. Fang, N. Yu, G. Yu, J. Huang, and C. M. Lieber, "Coaxial silicon nanowires as solar cells and nanoelectronic power sources," Nature, vol. 449, pp. 885, 2007. DOI: 10.1038/nature06181

[7] P. Kailuweit, M. Peters, J. Leene, K. Mergenthaler, F. Dimroth, and A. W. Bett, "Numerical simulations of absorption properties of InP nanowires for solar cell applications," Prog Photovolt Res Appl., vol. 20, no. 8, pp. 945-953, 2011. DOI: https://doi.org/10.1002/pip.1169

[8] V. Dhaka, T. Haggren, H. Jussila, H. Jiang, E. Kauppinen, T. Huhtio, M. Sopanen, and H. Lipsanen, "High Quality GaAs Nanowires Grown on Glass Substrates,” Nano Letters, vol. 12, no. 4, pp. 1912-1918, 2012. DOI: $10.1021 / \mathrm{n} 1204314 \mathrm{z}$

[9] E. Russo-Averchi, J. Vukajlovic Plestina, G. Tütüncüoglu, F. Matteini, A Dalmau-Mallorquí, M. de la Mata, D. Rüffer, H. A. Potts, J. Arbiol, S. Conesa-Boj, and A. Fontcuberta i Morral, "High Yield of GaAs Nanowire Arrays on Si Mediated by the Pinning and Contact Angle of Ga," Nano Letters, vol. 15, no. 5, pp. 2869-2874, 2015. DOI: 10.1021/n1504437v

[10] C. Gutsche, R. Niepelt, M. Gnauck, A. Lysov, W. Prost, C. Ronning, and F.-J. Tegude, "Direct Determination of Minority Carrier Diffusion Lengths at Axial GaAs Nanowire p-n Junctions," Nano Lett., vol. 12, no. 3, pp. 1453-1458, 2012. DOI: 10.1021/n1204126n

[11] M. Triplett, Y. Yang, F. Léonard, A. A. Talin, M. S. Islam, and D. Yu, "Long Minority Carrier Diffusion Lengths in Bridged Silicon Nanowires," Nano Lett., vol. 15, no. 1, pp. 523-529, 2015. DOI: 10.1021/n1503870u

[12] B. M. Kayes, "Radial pn junction, wire array solar cells," PhD Thesis, California Institute of Technology, California, U.S., 2009.

[13] B. M. Kayes, H. A. Atwater, and N. S. Lewis, "Comparison of the device physics principles of planar and radial p-n junction nanorod solar cells," J. Appl. Phys., vol. 97, no. 11, pp. 114302, 2005. DOI: https://doi.org/10.1063/1.1901835

[14] R. Parize, A. Katerski, I. Gromyko, L. Rapenne, H. Roussel, E. Kärber, E. Appert, M. Krunks, and V. Consonni, "ZnO/TiO2/Sb2S3 Core-Shell Nanowire Heterostructure for Extremely Thin Absorber Solar Cells," J. Phys. Chem. C, vol. 121, no. 18, pp. 9672-9680, 2017. DOI: 10.1021/acs.jpcc.7b00178

[15] E. Oksenberg, S. Martí-Sánchez, R. Popovitz-Biro, J. Arbiol, and E. Joselevich, "Surface-Guided Core-Shell ZnSe@ZnTe Nanowires as Radial p-n Heterojunctions with Photovoltaic Behavior," ACS Nano, vol. 11, no. 6, pp. 6155-6166, 2017. DOI: 10.1021/acsnano.7b02199

[16] G. Jia, B. Eisenhawer, J. Dellith, F. Falk, A. Thøgersen, and A. Ulyashin, "Multiple Core-Shell Silicon Nanowire-Based Heterojunction Solar Cells,” J. Phys. Chem. C, vol. 117, no. 2, pp. 1091-1096, 2013. DOI: $10.1021 / \mathrm{jp} 311047 \mathrm{k}$

[17] A. S. Togonal, M. Foldyna, W. Chen, J. X. Wang, V. Neplokh, M. Tchernycheva, J. Nassar, P. Roca i Cabarrocas, and Rusli, "Core-Shell Heterojunction Solar Cells Based on Disordered Silicon Nanowire Arrays," J. Phys. Chem. C, vol. 120, no. 5, pp. 2962-2972, 2016. DOI: 10.1021/acs.jpcc.5b09618

[18] M. M. Adachi, M. P. Anantram, and K. S. Karim, "Core-shell silicon nanowire solar cells," Sci. Rep., vol. 3, pp. 1546, 2013. DOI: https://doi.org/10.1038/srep01546

[19] D. van Dam, N. J. J. van Hoof, Y. Cui, P. J. van Veldhoven, E. P. A. M. Bakkers, J. Gómez Rivas, and J. E. M. Haverkort, "High-Efficiency Nanowire Solar Cells with Omnidirectionally Enhanced Absorption Due to Self-Aligned Indium-Tin-Oxide Mie Scatterers," ACS Nano, vol. 10, no. 12, pp. 11414-11419, 2016. DOI: 10.1021/acsnano.6b06874

[20] M. A. Green, Y. Hishikawa, E. D. Dunlop, D. H. Levi, J. Hohl-Ebinger, and A. W. Y. Ho-Baillie, "Solar cell efficiency tables (version 52)," Prog Photovolt Res Appl., vol. 26, no. 7, pp. 427-436, 2018. DOI: https://doi.org/10.1002/pip.3040

[21] M. Heurlin, "Growth of Semiconductor Nanowires for Solar Cell Applications,” Ph.D. Thesis, Lund University, Lund, Sweden, 2015.

[22] J. Wallentin, P. Wickert, M. Ek, A. Gustafsson, L. Reine Wallenberg, M. H. Magnusson, L. Samuelson, K. Deppert, and M. T. Borgström, "Degenerate p-doping of InP nanowires for large area tunnel diodes," Appl. Phys. Lett, vol. 99, no. 25, pp. 253105, 2011. DOI: https://doi.org/10.1063/1.3669697

[23] J. Wallentin, and M. T. Borgström, "Doping of semiconductor nanowires," J. Mat. Res., vol. 26, no. 17, pp. 2142-2156, 2011. DOI: https://doi.org/10.1557/jmr.2011.214

[24] G. Otnes, "III-V Nanowire Solar Cells: Growth and Characterization," $\mathrm{Ph} . \mathrm{D}$. Thesis, Lund University, Lund, Sweden, 2018.

[25] Y. Cui, J. Wang, S. R. Plissard, A. Cavalli, T. T. T. Vu, R. P. J. van Veldhoven, L. Gao, M. Trainor, M. A. Verheijen, J. E. M. Haverkort, and E. P. A. M. Bakkers, "Efficiency Enhancement of InP Nanowire Solar Cells by Surface Cleaning," Nano Lett., vol. 13, no. 9, pp. 4113-4117, 2013. DOI: $10.1021 / \mathrm{n} 14016182$

[26] M. Heurlin, O. Hultin, K. Storm, D. Lindgren, M. T. Borgström, and L. Samuelson, "Synthesis of Doped InP Core-Shell Nanowires Evaluated Using Hall Effect Measurements," Nano Lett., vol. 14, no. 2, pp. 749-753, 2014. DOI: $10.1021 / \mathrm{n} 1404039 \mathrm{~d}$

[27] J. Bullock, M. Hettick, J. Geissbühler, A. J. Ong, T. Allen, Carolin M. Sutter-Fella, T. Chen, H. Ota, E. W. Schaler, S. De Wolf, C. Ballif, A. Cuevas, and A. Javey, "Efficient silicon solar cells with dopant-free asymmetric heterocontacts," Nat. Energy, vol. 1, pp. 15031, 2016. DOI: https://doi.org/10.1038/nenergy.2015.31

[28] K. Masuko, M. Shigematsu, T. Hashiguchi, D. Fujishima, M. Kai, N. Yoshimura, T. Yamaguchi, Y. Ichihashi, T. Mishima, N. Matsubara, T. Yamanishi, T. Takahama, M. Taguchi, E. Maruyama, and S. Okamoto, "Achievement of More Than 25\% Conversion Efficiency With Crystalline Silicon Heterojunction Solar Cell," IEEE J. Photovolt., vol. 4, no. 6, pp. 1433-1435, 2014. DOI: https://doi.org/10.1109/JPHOTOV.2014.2352151

[29] T. Kato, A. Handa, T. Yagioka, T. Matsuura, K. Yamamoto, S. Higashi, J. Wu, K. F. Tai, H. Hiroi, T. Yoshiyama, T. Sakai, and H. Sugimoto, "Enhanced Efficiency of $\mathrm{Cd}$-Free $\mathrm{Cu}(\mathrm{In}, \mathrm{Ga})(\mathrm{Se}, \mathrm{S}) 2$ Minimodule Via (Zn,Mg)O Second Buffer Layer and Alkali Metal Post-Treatment," IEEE J. Photovolt., vol. 7, no. 6, pp. 1773-1780, 2017. DOI: 10.1109/JPHOTOV.2017.2745710

[30] W. S. Yang, J. H. Noh, N. J. Jeon, Y. C. Kim, S. Ryu, J. Seo, and S. I. Seok, "High-performance photovoltaic perovskite layers fabricated through intramolecular exchange," Science, vol. 348, no. 6240, pp. 1234, 2015. DOI: $10.1126 /$ science.aaa9272

[31] J. Yoon, S. Jo, I. S. Chun, I. Jung, H.-S. Kim, M. Meitl, E. Menard, X. Li, J. J. Coleman, U. Paik, and J. A. Rogers, "GaAs photovoltaics and optoelectronics using releasable multilayer epitaxial assemblies," Nature, vol. 465, pp. 329, 2010. DOI: https://doi.org/10.1038/nature09054

[32] M. A. Isik C. Kizilyalli, Harry Atwater, Thomas J. Gmitter, Gang He, Andreas Hegedus, Gregg Higashi, "Thin absorber layer of a photovoltaic device", US, U. S. Patent Office, 2014.

[33] V. Raj, T. S. dos Santos, F. Rougieux, K. Vora, M. Lysevych, L. Fu, S. Mokkapati, H. H. Tan, and C. Jagadish, "Indium phosphide based solar cell using ultra-thin $\mathrm{ZnO}$ as an electron selective layer," J. Phys. D Appl. Phys., vol. 51, no. 39, 2018. DOI: https://doi.org/10.1088/13616463/aad7e3

[34] X. Yin, C. Battaglia, Y. Lin, K. Chen, M. Hettick, M. Zheng, C.-Y. Chen, D. Kiriya, and A. Javey, "19.2\% Efficient InP Heterojunction Solar Cell with Electron-Selective TiO2 Contact," ACS Photonics, vol. 1, no. 12, pp. 1245-1250, 2014. DOI: 10.1021/ph500153c

[35] Lumerical Inc., https://www.lumerical.com/products/FDTD/

[36] R. A. M. Umran, S Inan, "Numerical Electromagnetics: The FDTD Method", United Kingdom: Cambridge University Press, 2011.

[37] W. Wang, X. Chi, Y. Feng and Y. Zhao, "Parallel FDTD Simulation of Photonic Crystals and Thin-Film Solar Cells," 13th International Conference on Parallel and Distributed Computing: Applications and Technologies, Beijing, pp. 773-776, 2012. DOI: 10.1109/PDCAT.2012.106

[38] N. Anttu, and H. Q. Xu, "Efficient light management in vertical nanowire arrays for photovoltaics," Opt. Express, vol. 21, no. S3, pp. A558-A575, 2013. DOI: https://doi.org/10.1364/OE.21.00A558

[39] H. J. Joyce, C. J. Docherty, Q. Gao, H. H. Tan, C. Jagadish, J. LloydHughes, L. M. Herz, and M. B. Johnston, "Electronic properties of GaAs, InAs and InP nanowires studied by terahertz spectroscopy," Nanotechnology, vol. 24, no. 21, 2013. DOI: 10.1088/0957$4484 / 24 / 21 / 214006$

[40] H. J. Joyce, J. Wong-Leung, C. K. Yong, C. J. Docherty, S. Paiman, Q. Gao, H. H. Tan, C. Jagadish, J. Lloyd-Hughes, L. M. Herz, and M. B. 
Johnston, "Ultralow Surface Recombination Velocity in InP Nanowires Probed by Terahertz Spectroscopy," Nano Lett., vol. 12, no. 10, pp. 53255330, 2012. DOI: $10.1021 / \mathrm{nl} 3026828$

[41] N. Anttu, K. L. Namazi, P. M. Wu, P. Yang, H. Xu, H. Q. Xu, and U. Håkanson, "Drastically increased absorption in vertical semiconductor nanowire arrays: A non-absorbing dielectric shell makes the difference," Nano Res., vol. 5, no. 12, pp. 863-874, 2012. DOI: https://doi.org/10.1007/s12274-012-0270-x

[42] Y. Yu, V. E. Ferry, A. P. Alivisatos, and L. Cao, "Dielectric Core-Shell Optical Antennas for Strong Solar Absorption Enhancement," Nano Lett., vol. 12, no. 7, pp. 3674-3681, 2012. DOI: 10.1021/nl301435r

[43] S.-K. Kim, X. Zhang, D. J. Hill, K.-D. Song, J.-S. Park, H.-G. Park, and J. F. Cahoon, "Doubling Absorption in Nanowire Solar Cells with Dielectric Shell Optical Antennas," Nano Lett., vol. 15, no. 1, pp. 753 758, 2015. DOI: $10.1021 / \mathrm{nl} 504462 \mathrm{e}$

[44] Z. Zhong, Z. Li, Q. Gao, Z. Li, K. Peng, L. Li, S. Mokkapati, K. Vora, J. Wu, G. Zhang, Z. Wang, L. Fu, H. H. Tan, and C. Jagadish, "Efficiency enhancement of axial junction InP single nanowire solar cells by dielectric coating," Nano Energy, vol. 28, pp. 106-114, 2016. DOI: https://doi.org/10.1016/j.nanoen.2016.08.032

[45] D. Wu, X. H. Tang, K. Wang, Z. B. He, and X. Q. Li, “An Efficient and Effective Design of InP Nanowires for Maximal Solar Energy Harvesting," Nanoscale Res. Lett., vol. 12, 2017. DOI: 10.1186/s11671017-2354-8.

[46] N. Anttu, "Physics and design for $20 \%$ and $25 \%$ efficiency nanowire array solar cells," Nanotechnology, vol. 30, no. 7, pp. 074002, 2018. DOI: https://doi.org/10.1088/1361-6528/aaf3f5

[47] N. Anttu, A. Abrand, D. Asoli, M. Heurlin, I. Aberg, L. Samuelson, and M. Borgstrom, "Absorption of light in InP nanowire arrays," Nano Res., vol. 7, no. 6, pp. 816-823, 2014. DOI: https://doi.org/10.1007/s12274014-0442-y

[48] Y. Chen, P. Kivisaari, M. E. Pistol, and N. Anttu, "Optimized efficiency in InP nanowire solar cells with accurate 1D analysis," Nanotechnology, vol. 29 , no. 4, 2018. DOI: 10.1088/1361-6528/aa9e73

[49] "Intrinsic Carrier Concentration," https://pveducation.org/pvcdrom/pnjunctions/intrinsic-carrier-concentration

[50] A. Cuevas, Y. Wan, D. Yan, C. Samundsett, T. Allen, X. Zhang, J. Cui, and J. Bullock, "Carrier population control and surface passivation in solar cells," Sol. Energy Mater Sol. Cells, vol. 184, pp. 38-47, 2018. DOI: https://doi.org/10.1016/j.solmat.2018.04.026

[51] U. Würfel, A. Cuevas, and P. Würfel, "Charge Carrier Separation in Solar Cells,” IEEE J. Photovolt., vol. 5, no. 1, pp. 461-469, 2015. DOI: 10.1109/JPHOTOV.2014.2363550

[52] Z. Li, Y. C. Wenas, L. Fu, S. Mokkapati, H. H. Tan, and C. Jagadish, "Influence of Electrical Design on Core-Shell GaAs Nanowire Array Solar Cells," IEEE J. Photovolt., vol. 5, no. 3, pp. 854-864, 2015. DOI: 10.1109/JPHOTOV.2015.2405753

[53] H. Goto, K. Nosaki, K. Tomioka, S. Hara, K. Hiruma, J. Motohisa, and T. Fukui, "Growth of Core-Shell InP Nanowires for Photovoltaic Application by Selective-Area Metal Organic Vapor Phase Epitaxy," Appl. Phys. Express, vol. 2, no. 3, 2009. https://doi.org/10.1143/APEX.2.035004

[54] N. Tajik, C. M. Haapamaki, and R. R. LaPierre, "Photoluminescence model of sulfur passivated p-InP nanowires," Nanotechnology, vol. 23, no. 31, pp. 315703, 2012. DOI: https://doi.org/10.1088/09574484/23/31/315703

[55] L. E. Black, A. Cavalli, M. A. Verheijen, J. E. M. Haverkort, E. P. A. M. Bakkers, and W. M. M. Kessels, "Effective Surface Passivation of InP Nanowires by Atomic-Layer-Deposited $\mathrm{Al} 2 \mathrm{O} 3$ with POx Interlayer," Nano Lett., vol. 17, no. 10, pp. 6287-6294, 2017. DOI: 10.1021/acs.nanolett.7b02972

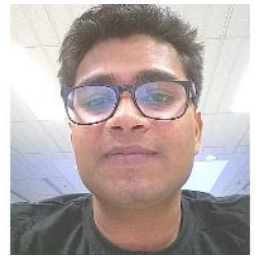

Vidur Raj (M'16) received his B.Tech+M.tech (Dual Degree) in nanotechnology from Amity Universty, Noida, India, in 2014. Currently, he is working toward his Ph.D. degree in physics under Prof. Hoe Tan, Prof. Lan Fu and Prof. Chennupati Jagadish at The Australian National University, Canberra, Australia. His topic of Ph.D. is "Optoelectronic Simulation and Fabrication of Thin Flim/Nanowire Heterojunction Solar Cells based on InP"

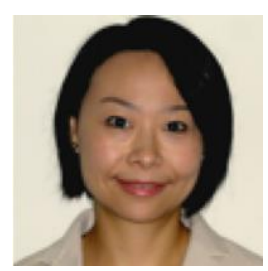

Lan Fu (SM'07) received the M.Sc. degree from the University of Science and Technology of China, Hefei, China, in 1996 and the Ph.D. degree from The Australia National University, Canberra, Australia, in 2001. She is currently a Professor the Department of Electronic Materials Engineering, The Australia National University. Her main research interests include the design, fabrication, and integration of optoelectronic devices (lasers and photodetectors) and high-efficiency solar cells based on lowdimensional III-V compound semiconductor structures, including quantum wells, self-assembled quantum dots, and nanowires grown by metal-organic chemical vapor deposition (MOCVD).

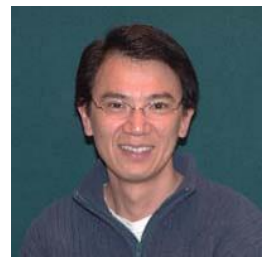

Hark Hoe Tan (SM'14-F'19) received the B.Eng. (Hons.) degree in electrical engineering from the University of Melbourne in 1992, after which he worked with Osram in Malaysia as a Quality Assurance Engineer. In 1997, he received the $\mathrm{PhD}$ degree from The Australian National University for his dissertation entitled \&quot;Ion beam effects in GaAs/AlGaAs material and devices." He has been the past recipient of the Australian Research Council Postdoctoral, QEII and Future Fellowships. He has published/co-published over 400 journal papers and 6 book chapters, and is a co-inventor in 4 US patents related to laser diodes and infrared photodetectors. His research interests include epitaxial growth of low-dimensional compound semiconductors, nanostructured optoelectronic devices and ionimplantation processing of compound semiconductors for optoelectronic device applications. Prof. Tan has been elected as a Fellow of the IEEE from 2019 and was the Distinguished Lecturer for IEEE Nanotechnology Council (2016 \&amp; 2017) and IEEE Photonics Society (2016-2017). He has been the past chair of the Photonics Society, Electron Devices Society and Nanotechnology Council chapters of the IEEE ACT section.

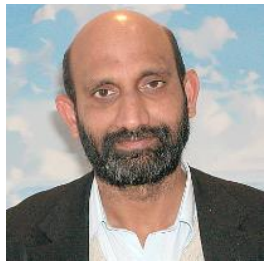

Chennupati Jagadish AC (F'02) is a Distinguished Professor and Head of Semiconductor Optoelectronics and Nanotechnology Group in the Research School of Physics and Engineering, Australian National University. He has served as Vice-President and Secretary Physical Sciences of the Australian Academy of Science during 
2012-2016. He is currently serving as President of IEEE Photonics Society, and President of Australian Materials Research Society. His research interests include optoelectronics nanotechnology and neuroscience. Prof. Jagadish is an Editor/Associate editor of 6 Journals (EIC: Progress in Quantum Electronics), 3 book series and serves on editorial boards of 20 other journals. He has published more than 900 research papers (620 journal papers), holds 5 US patents, co-authored a book, co-edited 12 books and edited 12 conference proceedings and 17 special issues of Journals. He is a Fellow of 8 Science and Engineering Academies (the Australian Academy of Science, Australian Academy of Technological Sciences and Engineering, The World Academy of Sciences, US National Academy of Inventors, Indian National Science Academy (Foreign Fellow), Indian National Academy of Engineering (Foreign Fellow), Indian Academy of Science (Honorary Fellow), Andhra Pradesh Akademi of Sciences (Honorary Fellow)) and a Fellow of 14 Professional Societies (IEEE, APS, MRS, OSA, AVS, ECS, SPIE, AAAS, FEMA, APAM, IoP (UK), IET (UK), IoN (UK) and the AIP). He received Peter Baume Award from the ANU in 2006, the Quantum Device Award from ISCS in 2010, Electronics and Photonics Division Award of the Electrochemical Society in 2012, 2013 Walter Boas Medal, 2015 IEEE Pioneer Award in Nanotechnology, 2015 IEEE Photonics Society Engineering Achievement Award, 2016 MRSI Silver Jubilee Anniversary Medal, 2016 Distinguished Fellow of Chinese Academy of Sciences, 2016 OSA Nick Holonyak Award, 2017 Welker Award, 2017 IUMRS Somiya Award, 2017 Nayudamma Award, 2018 AVS Nanotechnology Recognition Award and 2018 UNESCO Medal for contributions to the development of $\underline{\text { nanoscience and nanotechnologies. He has received Australia's }}$ highest civilian honor, AC, Companion of the Order of Australia in 2016 for his contribution to Physics and Engineering, in particular Nanotechnology. 

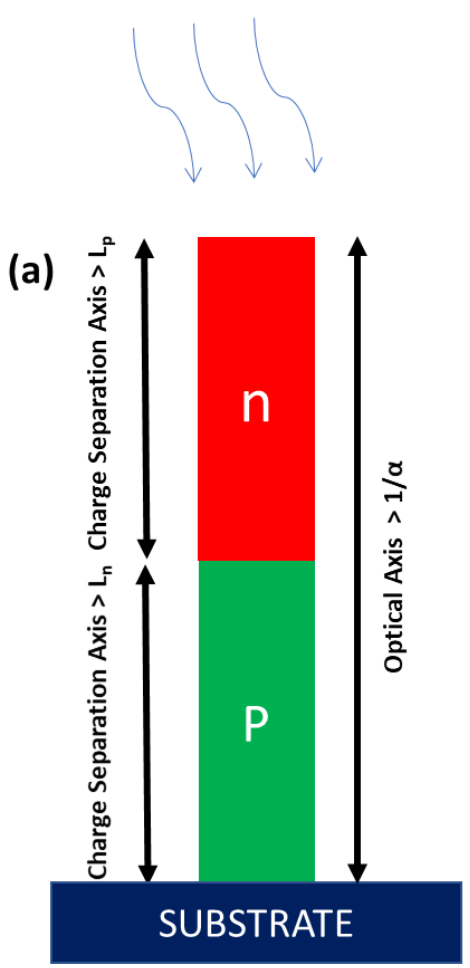

(b)

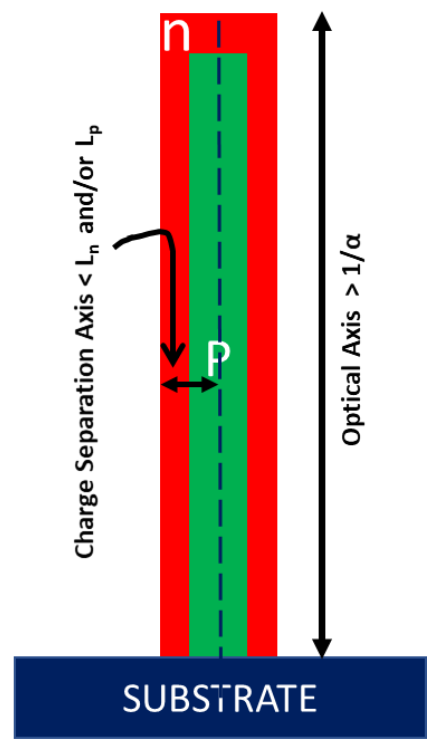

Fig. 1. Schematic representation of (a) an axial p-n junction nanowire solar cell and (b) a radial p-n junction nanowire solar cell. In both of these figures, $\alpha$ denotes the absorption coefficient of the active material and $L_{n}$ and $L_{p}$, denotes the electrons and holes diffusion lengths, respectively.

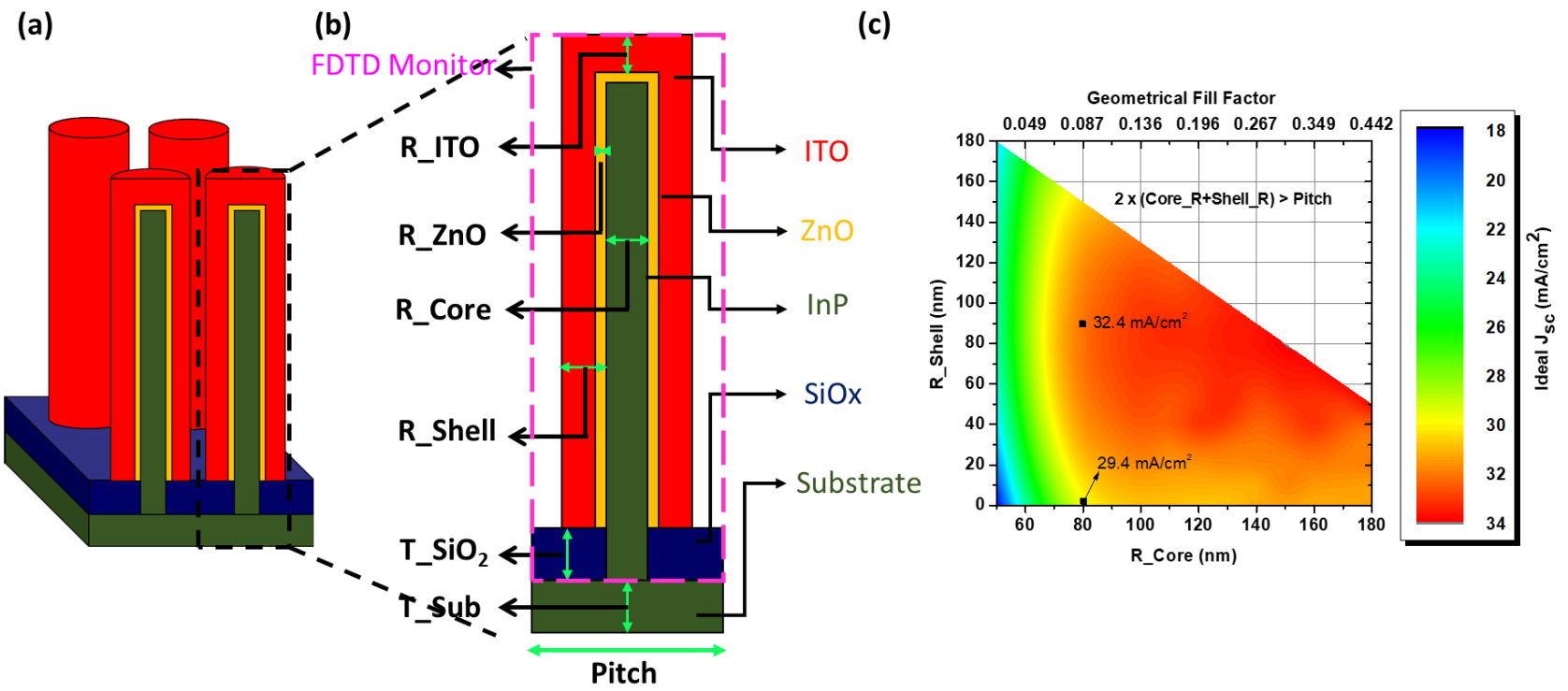

Fig. 2. (a) Schematic illustration of an array of radial p-n heterojunction solar cells based on InP. (b) Enlarged 2-D cross-section schematic of the solar call shown in Figure 1(a), along with material specifications and geometrical parameters. Thickness (radius) of $\mathrm{ZnO}$, ITO and InP core, is denoted by R_ITO, R_ZnO, and R_Core, respectively. Total thickness of shell is measured as the sum of the thickness of $\mathrm{ZnO}$ and ITO and is denoted by R_Shell. The thickness of substrate and silicon dioxide is denoted as $\mathrm{T}_{-} \mathrm{Sub}$ and $\mathrm{T}-\mathrm{SiO}_{2}$, respectively. (c) Ideal short current calculated for InP nanowire array of $480 \mathrm{~nm}$ pitch as a function of core radius (R_Core) and shell thickness (R_Shell). In above figure, the top axis shows the geometrical fill factor and the two points marked in black square on the plot shows the $\mathbf{J}_{\text {sc(ideal }}$ before and after oxide coating. 

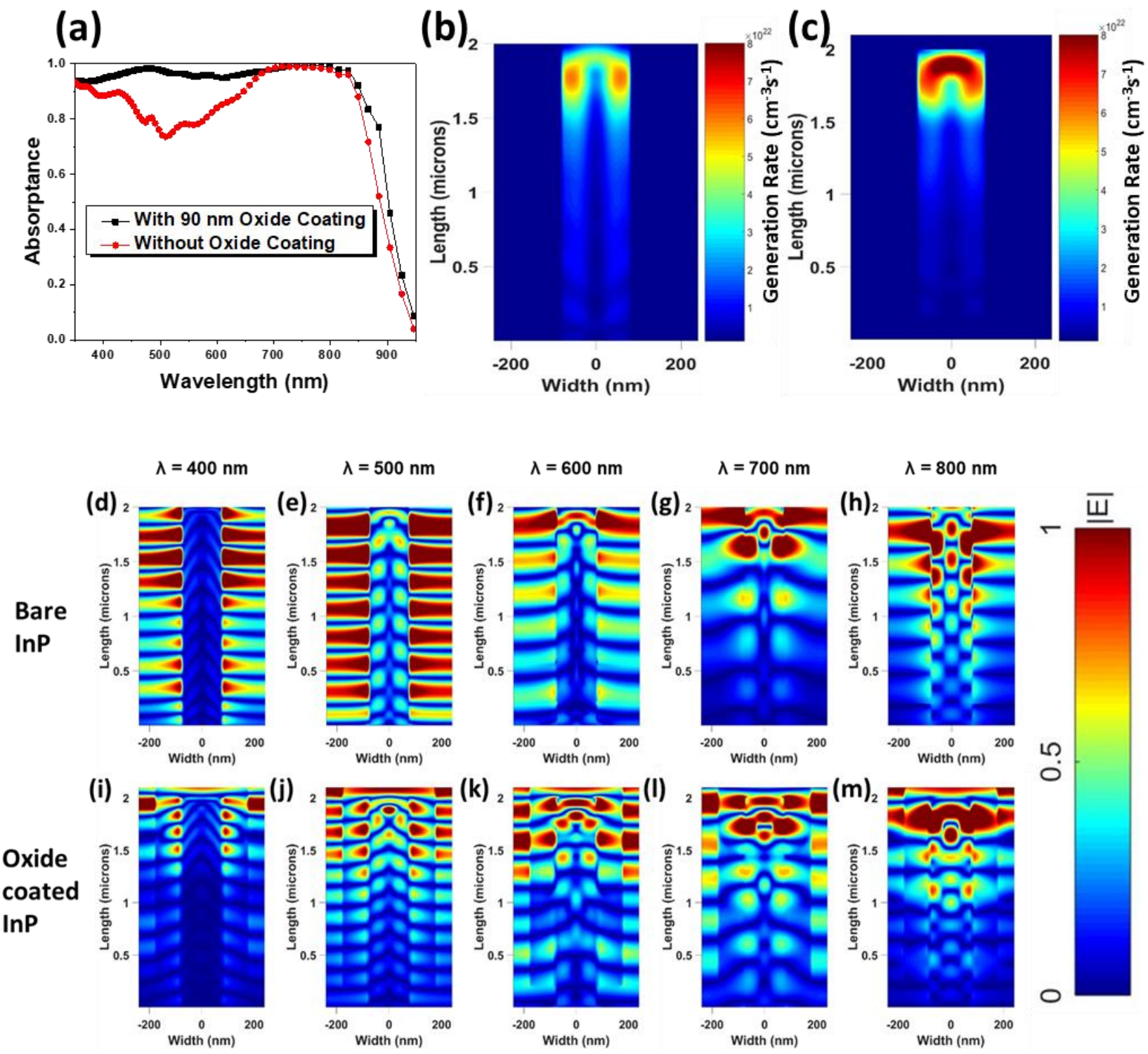

Fig. 3. (a) Comparative simulated absorption spectra for $\mathrm{InP}$ core with $80 \mathrm{~nm}$ radius, coated with and without a $90 \mathrm{~nm}$ wide shell $(10 \mathrm{~nm} \mathrm{ZnO}+80 \mathrm{~nm}$ ITO). Simulated optical generation rate of electrons and holes pairs in the y-z cross section of (b) only InP and (c) InP core with $90 \mathrm{~nm}$ oxide, respectively. The normalized electric field distribution in a bare $\mathrm{InP}$ of radius $80 \mathrm{~nm}$ at wavelength (d) $400 \mathrm{~nm}$, (e) $500 \mathrm{~nm}$, (f) $600 \mathrm{~nm}$, (g) $700 \mathrm{~nm}$ and (h) $800 \mathrm{~nm}$, respectively. The normalized electric field distribution in an $\mathrm{nP}$ core of radius $80 \mathrm{~nm}$ with a $90 \mathrm{~nm}$ thick oxide shell at wavelength (i) 400 $\mathrm{nm}(\mathrm{j}) 500 \mathrm{~nm}(\mathrm{k}) 600 \mathrm{~nm}(1) 700 \mathrm{~nm}$ and (m) 800nm, respectively. 


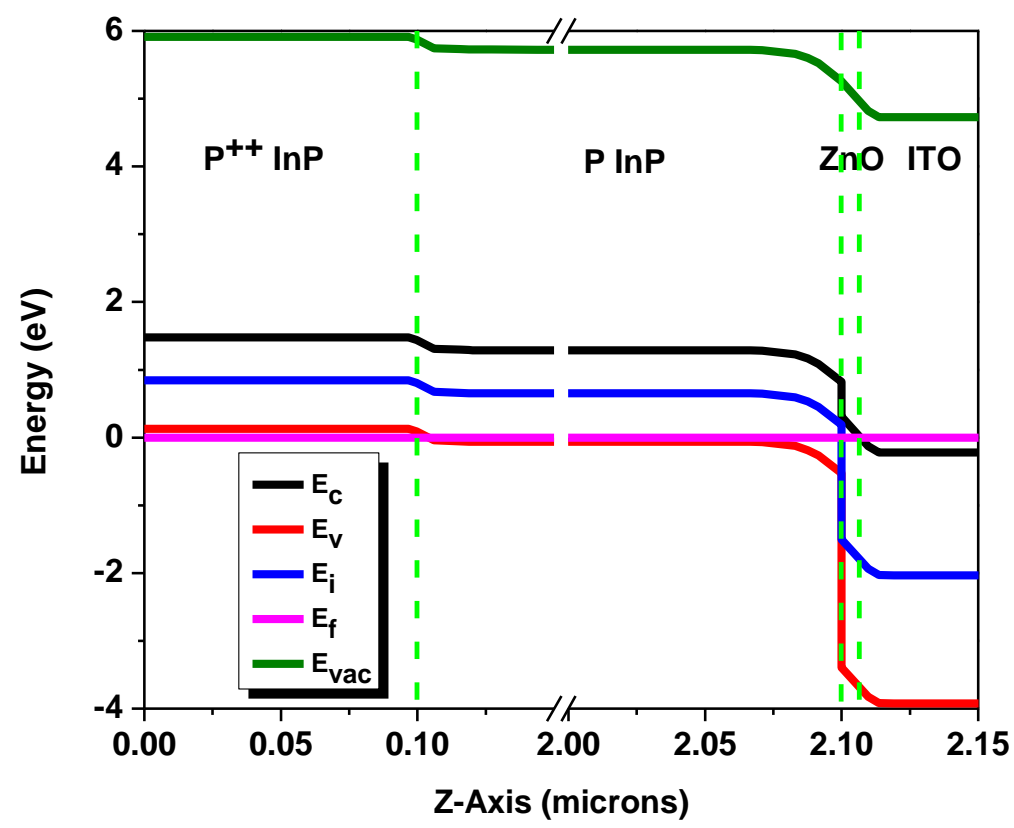

Fig. 4. Band diagram of a heterojunction solar cell along z-axis at thermal equilibrium.
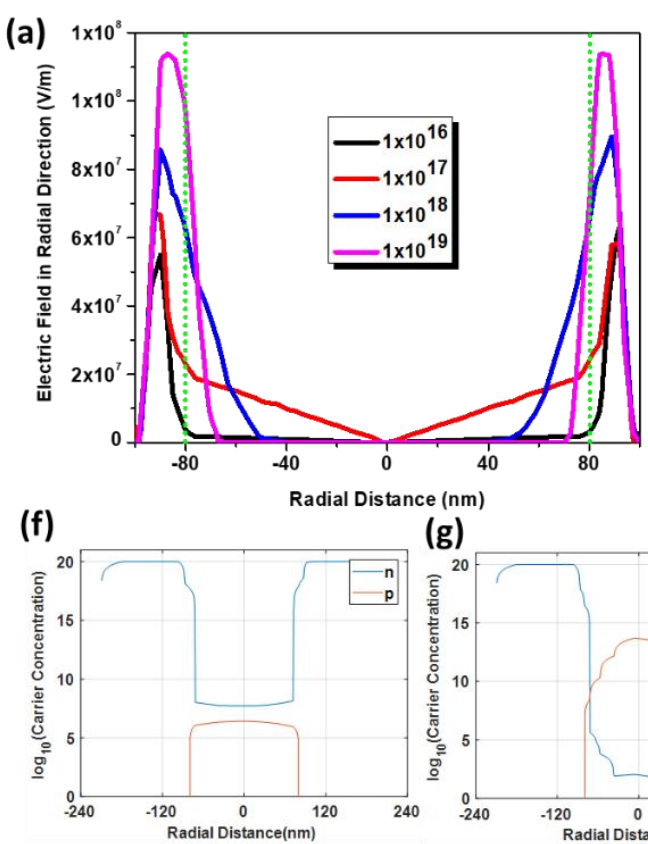

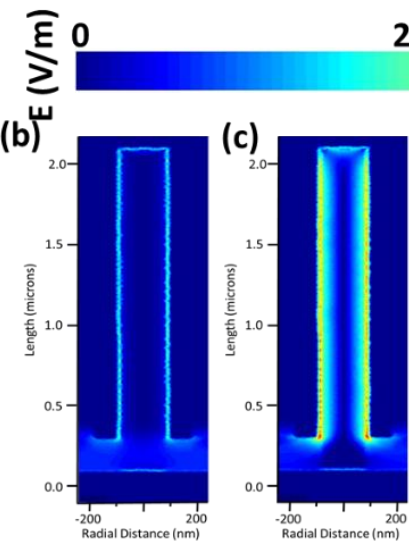

(h)
$2.5 \times 10^{7}$
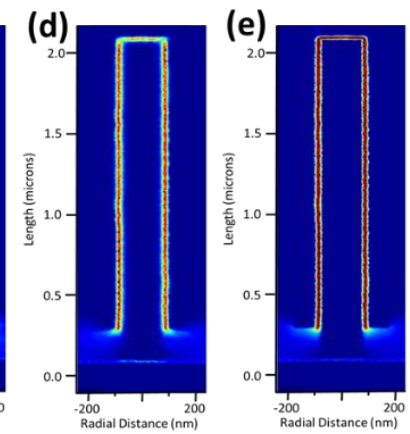

(i)

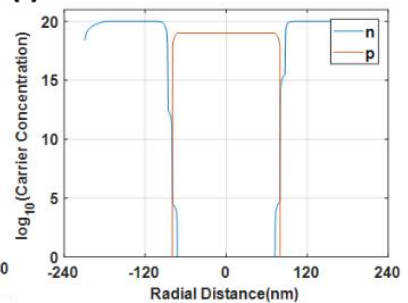

Fig. 5. (a) Electric field profile plotted against radial distance. 2-D plot of simulated electric field for ITO/ZnO/p-InP heterojunction at thermal equilibrium when the p-type doping concentration in core is (b) $1 \times 10^{16} \mathrm{~cm}^{-3}$, (c) $1 \times 10^{17} \mathrm{~cm}^{-3}$, (d) $1 \times 10^{18} \mathrm{~cm}^{-3}$, and (e) $1 \times 10^{19} \mathrm{~cm}^{-3}$. Corresponding simulated charge carrier concentration as a function of radial distance is plotted for core doping concentration of $(\mathrm{f}) 1 \times 10^{16} \mathrm{~cm}^{-3},(\mathrm{~g}) 1 \times 10^{17} \mathrm{~cm}^{-3},(\mathrm{~h})$ $1 \times 10^{18} \mathrm{~cm}^{-3}$, and (i) $1 \times 10^{19} \mathrm{~cm}^{-3}$. 

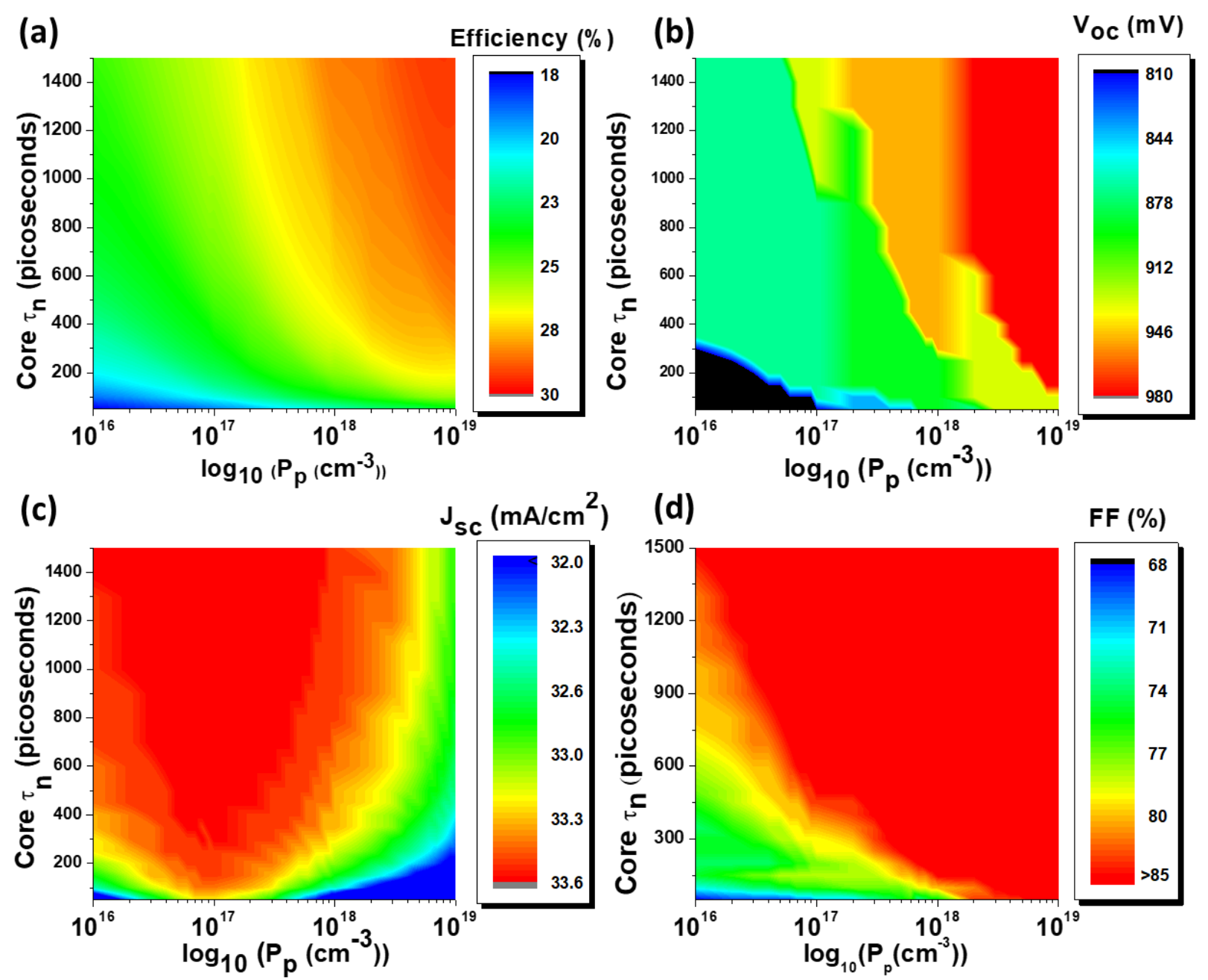

Fig. 6. Solar cell performance parameters are plotted as a function of core minority carrier lifetime ( $\left.\boldsymbol{\tau}_{\boldsymbol{n}}\right)$ and p-type core doping concentration (in absence of surface recombination at $\mathrm{ZnO} / \mathrm{InP}$ interface): (a) Efficiency (b) $\mathrm{V}_{\mathrm{oc}}$ (c) $\mathrm{J}_{\mathrm{sc}}$ and (d) FF. 


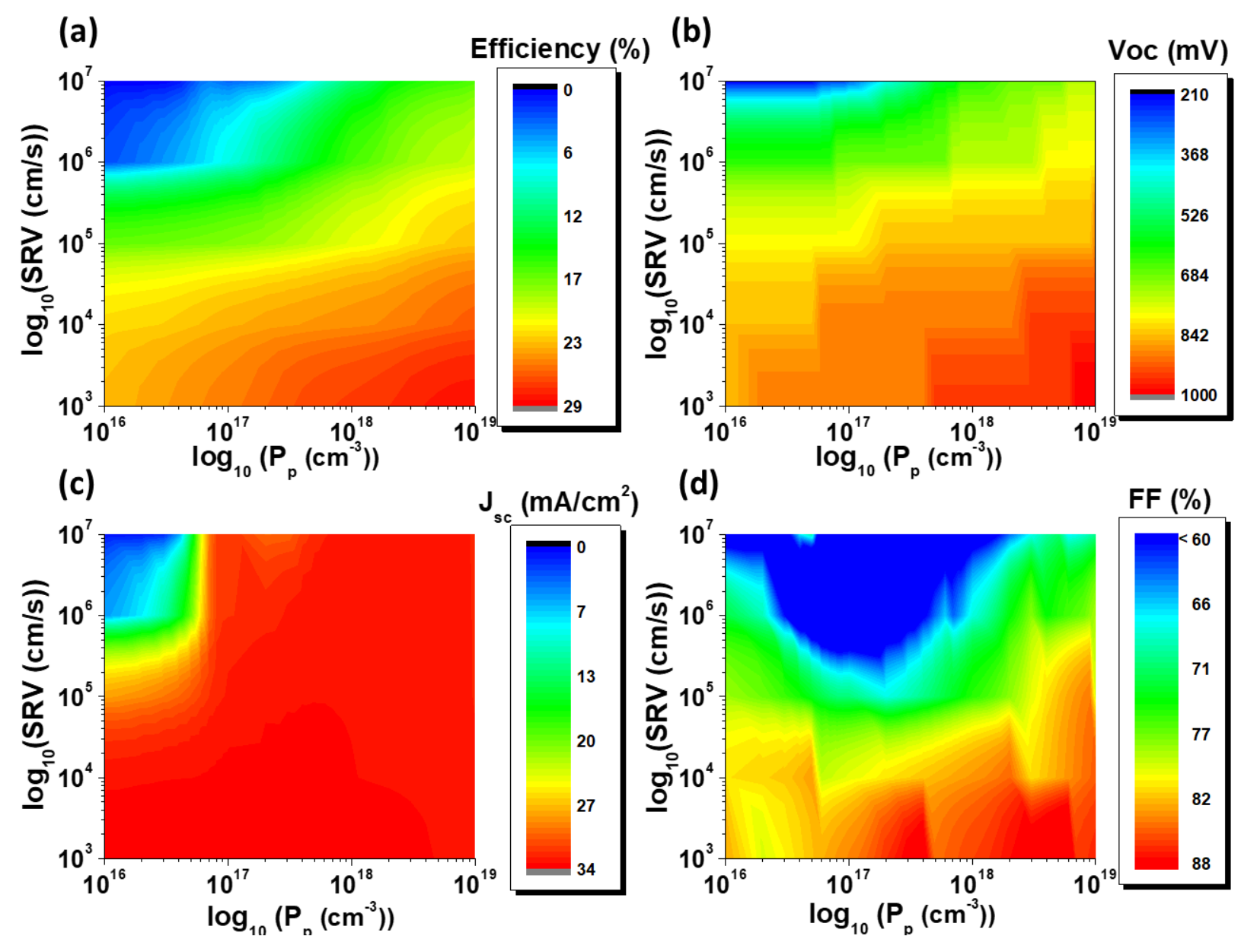

Fig. 7. Effect of SRV (at $\mathrm{ZnO} / \mathrm{InP}$ interface) on solar cell performance parameters are plotted as a function of p-type core doping concentration for $\boldsymbol{\tau}_{\boldsymbol{n}}=\mathbf{1} \boldsymbol{n s}$ (a) Efficiency (b) $\mathrm{V}_{\mathrm{oc}}$ (c) $\mathrm{J}_{\mathrm{sc}}$ and (d) FF. 


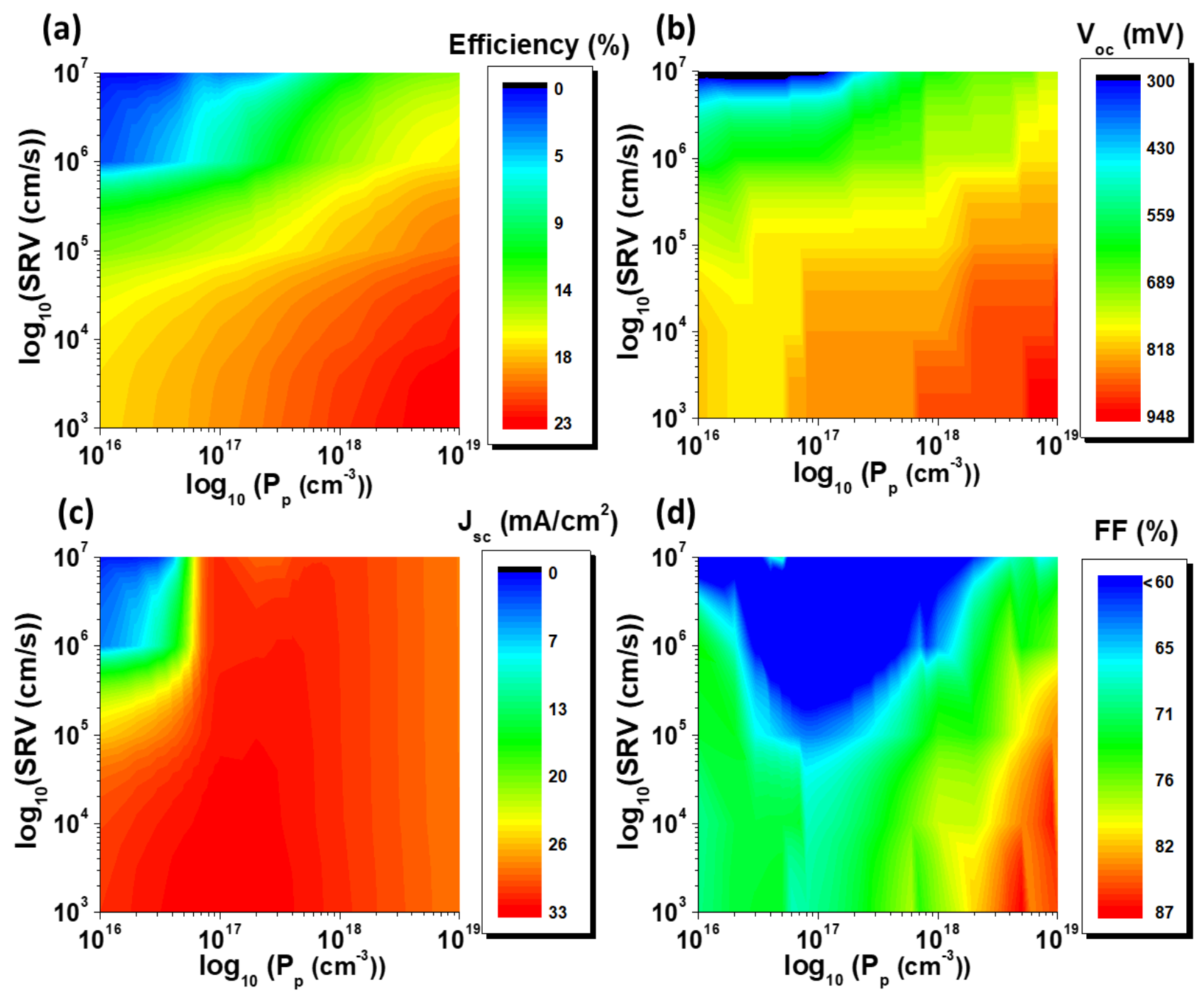

Fig. 8. Effect of SRV (at $\mathrm{ZnO} / \mathrm{InP}$ interface) on solar cell performance parameters are plotted as a function of p-type core doping concentration for

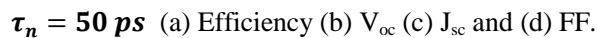

